Douglas M. Smith, MD

Arastoo Vossough, MD, $\mathrm{PhD}$

Gregory A. Vorona, MD

Lauren A. Beslow, MD, MSCE

Rebecca N. Ichord, MD

Daniel J. Licht, MD

Correspondence to

Dr. Smith:

smithd13@email.chop.edu

Supplemental data at Neurology.org

\section{Pediatric cavernous sinus thrombosis}

\author{
A case series and review of the literature
}

[미

\section{ABSTRACT}

Objective: To describe clinical characteristics, imaging findings, morbidity, and mortality in a single-center cohort of 12 pediatric cavernous sinus thrombosis cases and to review all cases available in recent English literature.

Methods: Clinical data and radiographic studies on 12 cases from our institution were analyzed retrospectively. A literature search and review was conducted, with additional cases pooled with the new cohort for an aggregate analysis.

Results: Twelve cases of cavernous sinus thrombosis in children from the Children's Hospital of Philadelphia between January 1, 2000, and December 31, 2013, were reviewed. All patients survived to discharge; 3 of 12 (25\%) experienced neurologic morbidity. Contrast-enhanced MRI and contrast-enhanced head CT were $100 \%$ sensitive for detecting cavernous sinus thrombosis, while noncontrast time-of-flight magnetic resonance venography (TOF MRV) and noncontrast head CT were $0 \%$ sensitive. Literature review produced an additional 40 cases, and the aggregate mortality rate was 4 of $52(8 \%)$ and morbidity rate was 10 of 40 (25\%). Outcomes did not vary by treatment or with unilateral vs bilateral cavernous sinus involvement. There was a trend toward worse outcomes with fungal infections.

Conclusion: Our case series demonstrates low morbidity and mortality with early, aggressive surgical, antimicrobial, and anticoagulation therapies. Although anticoagulation and surgery were not associated with significantly different outcomes, more study is needed. Neurology ${ }^{\circledR} 2015 ; 85: 763-769$

\section{GLOSSARY}

CST = cavernous sinus thrombosis; ICA = internal carotid artery; $\mathbf{M R A}=$ magnetic resonance angiography; $\mathbf{M R V}=$ magnetic resonance venography; TOF MRV = time-of-flight magnetic resonance venography without contrast.

Cavernous sinus thrombosis (CST) is a rare, life-threatening infectious complication involving the cavernous sinus. Although rarely seen in children, case reports have described it as a complication associated with septic thrombosis of the internal jugular vein, ${ }^{1}$ bacterial meningitis, ${ }^{2}$ sinusitis, ${ }^{3}$ otitis media, ${ }^{4}$ dental abscess, ${ }^{5}$ facial soft-tissue infections, ${ }^{6}$ wasp bites, ${ }^{7}$ and other causes. During the preantibiotic era, mortality was estimated to be $100 \%,{ }^{8}$ and was found to be $79 \%$ as recently as $1988 .{ }^{9}$ In adults, mortality estimates approach $30 \% .{ }^{10}$ Morbidity can be severe because injury to the contents of the cavernous sinus can result in stroke, ophthalmoplegia, and blindness.

Due to its rarity, there are limited data available in children regarding CST clinical presentation, treatment, and prognosis. The largest case series, published in India, included 14 children from 1985 to 1988 ; 11 of these children died, and the 3 survivors had permanent deficits. ${ }^{11}$ More recently, a cohort in Thailand published in 2001 including 8 children over a 30year time period ${ }^{12}$ found favorable outcomes in 7 . The purpose of this study is to describe clinical and radiologic features, diagnostic evaluation, management, and prognosis of this disease in a single-center consecutive cohort. We analyzed data pooled from our study with cases found in a comprehensive review of childhood CST in the modern English literature.

From the Division of Neurology (D.M.S., R.N.I., D.J.L.) and the Department of Radiology (A.V.), Children's Hospital of Philadelphia, PA; the Department of Neurology (D.M.S., R.N.I., D.J.L.), Perelman School of Medicine of the University of Pennsylvania; the Department of Radiology (A.V.), Hospital of the University of Pennsylvania, Philadelphia; Children's Hospital of Richmond at VCU (G.A.V.), VA; and the Departments of Pediatrics and Neurology (L.A.B.), Yale University School of Medicine, New Haven, CT.

Go to Neurology.org for full disclosures. Funding information and disclosures deemed relevant by the authors, if any, are provided at the end of the article. 
METHODS Patient ascertainment was performed by querying the institution stroke registry database and the Picture Archiving and Communication System archive at the Children's Hospital of Philadelphia for cases between January 1, 2000, and December 31,2013 . The institution stroke database prospectively enrolled all confirmed stroke cases since 2006. This database was queried separately using the terms "cavernous sinus thrombosis" and "venous sinus thrombosis." All resulting cases were reviewed by one reviewer (D.M.S.) and all selected cases were confirmed by a second reviewer (D.J.L.). Radiologic studies were reviewed by 2 neuroradiologists (G.A.V., A.V.) by consensus. Patients were included if both reviewers found radiographic evidence of CST, as defined by the presence of venous vascular filling defects and lack of flow in the cavernous sinuses.

Standard protocol approvals, registrations, and patient consents. All children enrolled in the stroke registry database were consented for the use of their information. This study was reviewed and approved by Children's Hospital of Philadelphia's Institutional Review Board.

An electronic literature search was performed in PubMed to find relevant articles published between January 1, 1994, and November 1, 2014. Articles were restricted to those published in the English language. The search was performed using the search terms "cavernous sinus thrombosis" in all fields and "child*" or "pediatric" in all fields. The full-text articles included from the results of this search were reviewed by a single investigator (D.M.S.). Identifiable cases of CST in children between 1 and 17 years were abstracted for etiology, management, and outcomes. Cases were excluded if specific outcomes were not available or if a clinical diagnosis of CST was made but radiologic studies did not support the diagnosis.

Cases identified by literature review were abstracted and pooled with the new case series in aggregate.

RESULTS Query of the stroke database identified 8 patients. A search of Department of Radiology records identified 4 additional patients. These records were reviewed, and all 12 were included. Ages ranged from 3 to 16 years, with a mean of 10.6 years (SD 4.3 years). Seven patients (58\%) were male. Four patients $(33 \%)$ had evidence of bilateral CST. Time to presentation of symptoms attributable to CST was variable and for many patients duration was unclear. While 10 of 12 patients had nonspecific headache and fever, only half had symptoms directly attributable to cranial neuropathies from CST. Table 1 summarizes the 12 cases from our study.

Initial cranial imaging was $\mathrm{CT}$ for 10 of the 12 . Of these, 3 were noncontrast head CTs, and 7 were contrast-enhanced. None of the 3 noncontrast head CTs identified CST, although one did identify subtle enlargement of the cavernous sinus. All 7 of the contrast-enhanced head CTs identified CST when it was present (figure 1). All patients had contrastenhanced MRI. Additionally, 8 of 12 had time-of-flight magnetic resonance venography without contrast (TOF MRV); none of the TOF MRVs identified CST, even upon retrospective secondary review. On magnetic resonance angiography (MRA) imaging, 10 of $12(83 \%)$ had an abnormality of the internal carotid artery (ICA), which varied from mild narrowing to complete occlusion (figure 2). Despite the severity of these findings, there were no large arterial strokes. Punctate foci of restricted diffusion were seen in 4 of $12(33 \%)$ patients; none of these resulted in neurologic deficits. On follow-up imaging, all 10 cases of ICA narrowing had improved, and 7 of 10 (70\%) ICA abnormalities resolved entirely within 6 months.

Thrombosis of the superior ophthalmic vein was diagnosed in 3 of $12(25 \%)$ patients. Of these, none had decreased visual acuity at presentation. One patient complained of diplopia but did not have a detectable cranial nerve palsy on physical examination. Long-term, all of these patients experienced resolution of their symptoms with no lasting morbidity related to the disease.

Sinusitis was the suspected etiology in 11 of 12 patients $(92 \%)$, and all 11 of these patients underwent surgical drainage. Five of 12 (42\%) had intracranial purulent collections adjacent to the sphenoid sinus and cavernous sinuses. Only 2 of $12(17 \%)$ had a second distant intracranial focus of infection; one was a cerebellar abscess that presented a week after her initial presentation of CST and the other was a focus of cerebellar peduncle cerebritis and abscess. Neither patient experienced lasting morbidity. Of the $4 \mathrm{pa}-$ tients who underwent CSF analysis, 3 (75\%) had CSF pleocytosis with leukocyte counts between 1,300 and 4,458 cells per high-power field. All CSF cultures were sterile. Septic thrombophlebitis (Lemierre syndrome) of the internal jugular vein occurred in 2 of $12(17 \%)$ patients. Fusobacterium was not cultured from either of these patients. Two patients without Lemierre syndrome were diagnosed with Fusobacterium.

All patients were treated immediately with antibiotics that included coverage for Staphylococcus, Streptococcus, and anaerobes. Surgical debridement was performed in 11 of 12 (92\%); functional endoscopic sinus surgery was performed in 10 and myringotomy in 1. Therapeutic anticoagulation was initiated in 10 of 12 patients; all 10 had abnormalities of the internal carotid artery. In both patients who were not anticoagulated, there was a nonocclusive thrombus in the cavernous sinus in the presence of intracranial disease necessitating significant surgery. Median duration of anticoagulation was 3 months; $80 \%$ of those on anticoagulation were treated with low-molecular-weight heparin. There were no bleeding complications observed.

Outcome data were available for 11 of $12(92 \%)$ at a median interval of 11 months (interquartile range 2-30 months) after hospital discharge. All 12 patients survived to discharge. While 6 of $12(50 \%)$ patients 
Table 1 Summary of 12 cases of pediatric cavernous sinus thrombosis at The Children's Hospital of Philadelphia

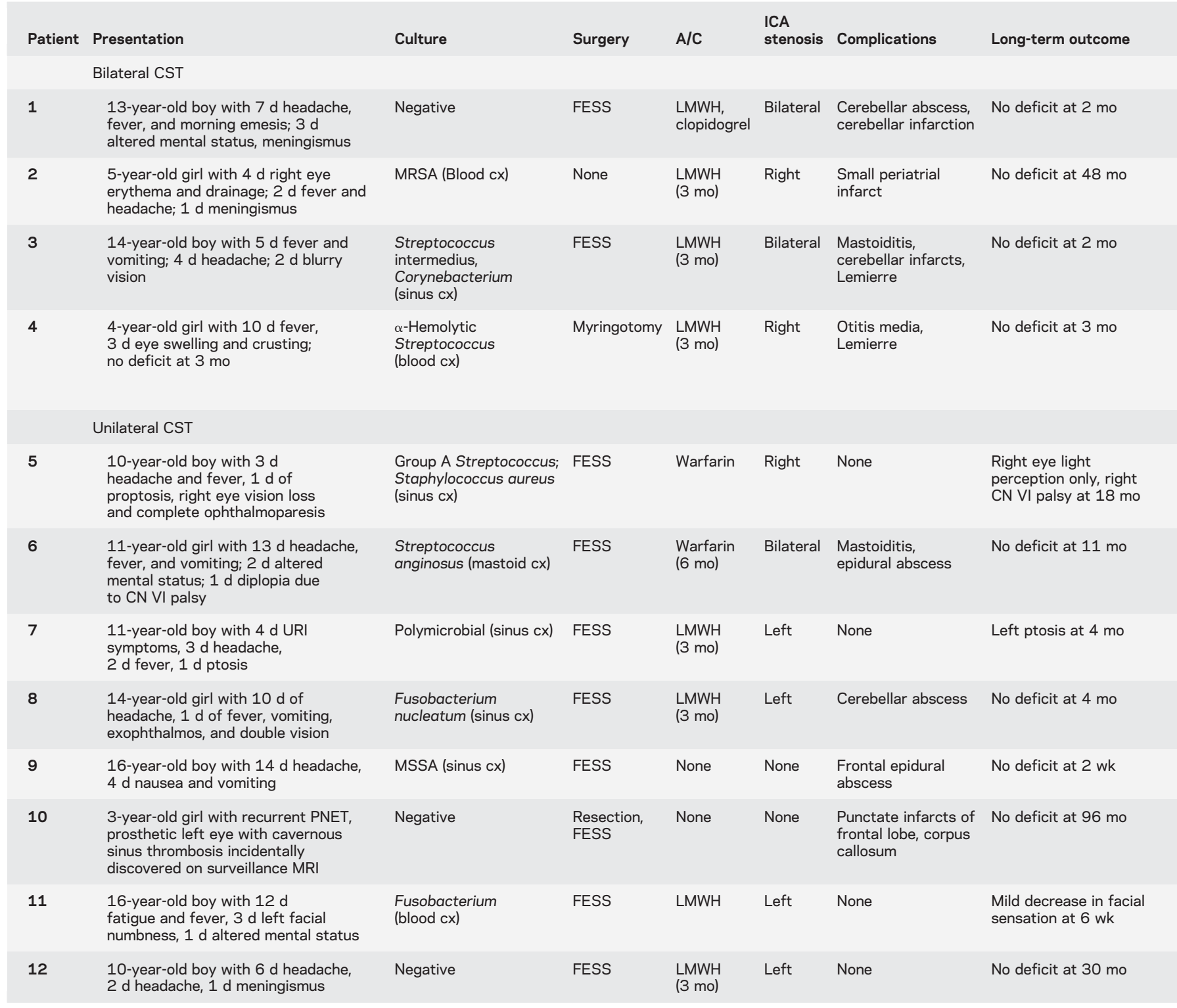

Abbreviations: $\mathrm{A} / \mathrm{C}=$ anticoagulation; $\mathrm{CN} \mathrm{VI}=$ cranial nerve $\mathrm{VI} ; \mathrm{CST}=$ cavernous sinus thrombosis; $\mathrm{cx}=$ culture; FESS = functional endoscopic sinus surgery; ICA = internal carotid artery; LMWH = low molecular weight heparin; MRSA = methicillin-resistant Staphylococcus aureus; MSSA = methicillinsensitive Staphylococcus aureus; PNET = primitive neuroectodermal tumor; URI = upper respiratory infection.

had cranial nerve palsies at presentation, only 3 had persistent symptoms at follow-up (one with persistent subtle ptosis, one with subtle sensory changes in a V1 and V2 distribution, one with monocular vision loss).

Literature review. Literature review results are summarized in figure 3. The initial PubMed search produced a total of 67 abstracts. Of these, 19 had no cases of CST. The remaining 48 articles were reviewed, which described a total of 88 cases of CST. In 7 cases, it was not possible to ascertain whether the patient survived, and these were excluded. Thirty-five cases either were outside the designated age parameters or did not specify the age of the patient. In 6 cases, the described radiographic findings were inconsistent with CST; alternative diagnoses included neoplastic changes of the cavernous sinus, craniopharyngioma, and clinical CST without changes within or adjacent to the cavernous sinuses. The remaining 40 cases of CST were included in the aggregate analysis of mortality in conjunction with the cases from our study for a total of 52 cases. Eight of the cases did not have clear long-term outcome data, and these were excluded from the aggregate morbidity analysis. A summary of all 52 cases is presented in table e- 1 on the Neurology ${ }^{\circledR}$ Web site at Neurology.org.

Overall, mortality was 4 of 52 (8\%). The first case resulting in mortality from CST was a Rhizomucor infection in a patient undergoing chemotherapy for 

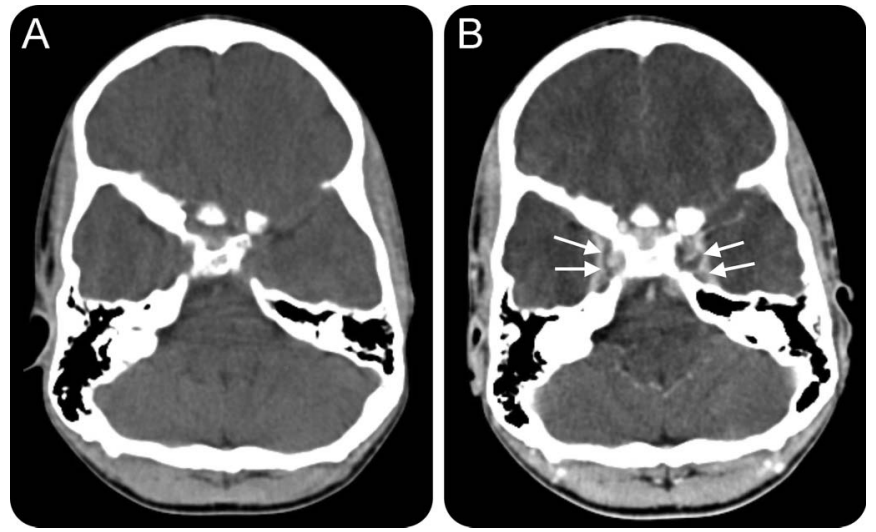

(A) Axial non-contrast-enhanced CT through the cavernous sinuses did not identify cavernous sinus thrombosis. This same patient had a repeat head CT performed with contrast (B), which shows multiple filling defects (white arrows) in both cavernous sinuses indicative of cavernous sinus thrombosis.

leukemia. ${ }^{13}$ There was a surgical procedure attempted on this patient and anticoagulation was not utilized. The second fatal case of CST was in a child with streptococcal bacterial meningitis and bacteremia. ${ }^{14}$ She received antibiotics but no anticoagulation or surgical intervention. In this case, the cause of death was unclear. In both of these cases, diabetes insipidus occurred late in the course. In the final 2 fatal cases, no data were available regarding etiology or management. ${ }^{15}$

In considering morbidity, 8 cases were excluded from analysis due to inadequate follow-up details. In all cases, survival to discharge was confirmed but it was unclear if any neurologic deficits persisted.

Figure 2 Internal carotid artery stenosis in cavernous sinus thrombosis

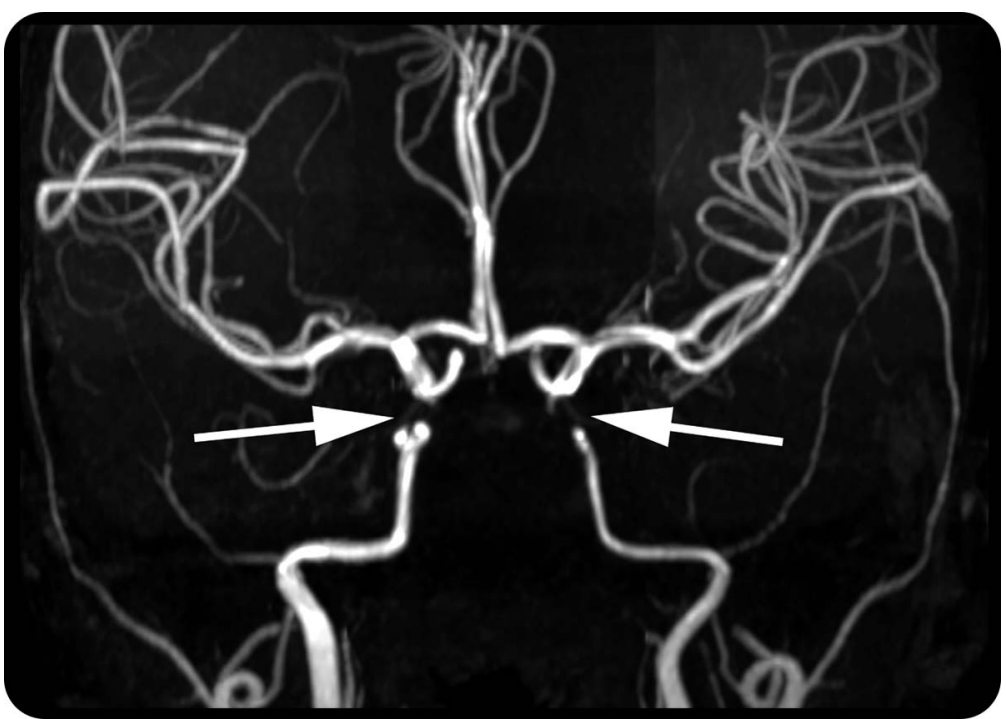

Magnetic resonance angiography shows severe narrowing of the bilateral cavernous segments of the internal carotid arteries (arrows) in a patient with bilateral cavernous sinus thrombosis.
Of the remaining 40 survivors with follow-up data available, $10(25 \%)$ experienced some degree of neurologic morbidity; however, only 6 (15\%) had a serious disability, characterized by either monocular blindness or hemiparesis.

In the aggregate series, 17 (45\%) had bilateral CST and 21 (55\%) had unilateral CST; in 14 cases, there was no information about whether CST was unilateral or bilateral. Six of $16(38 \%)$ children with bilateral CST experienced morbidity or mortality compared with 7 of 21 (33\%) with unilateral CST. In 3 of the 4 cases of fatal disease, the CST was bilateral.

Table 2 summarizes causative microbial agents. The most common cause in this series was Staphylococcus aureus, cultured in $24 \%$ of all cases. Multiple fungal species were cultured, and overall they represented $7 \%$ of cases. Despite their rarity, the 3 reported cases of CST associated with positive fungal cultures had very high morbidity and mortality, including one death and 2 cases of impaired vision. All cases of fungal CST were associated with comorbidities like acute lymphoblastic leukemia, ${ }^{13}$ recent steroid use, ${ }^{16}$ and poorly controlled diabetes. ${ }^{17}$ No other culture result seemed to be associated with outcome.

In the aggregate series, there was variation in the use of anticoagulation, although the majority of patients (54\%) were not anticoagulated. There did not seem to be a difference in outcome related to anticoagulation use, but the majority of cases in the literature did not comment on ICA abnormalities. Surgical intervention was utilized in 22 of 38 $(58 \%)$. In 14 cases, it was not specified whether surgery was performed. The majority $(15 / 22 ; 68 \%)$ underwent sinus surgery. Seven of $22(32 \%)$ who underwent surgery experienced morbidity, compared with 5 of $13(38 \%)$ who did not.

DISCUSSION Septic thrombosis of the cavernous sinus has been recognized since the early 19 th century as presenting a high risk of death or serious neurologic morbidity. Its frequency has been dramatically reduced since the advent of antibiotics, to the point that one otolaryngologist commented in 1977 that "everyone knows that [cavernous sinus thrombosis] is no longer a real clinical problem." ${ }^{\prime \prime}$ In the same publication, he found the mortality associated with this infection in adults to be $13.6 \%$ with long-term morbidity in $22.7 \%$ of survivors.

Comparable estimates of morbidity and mortality were previously not available in the pediatric literature. This study is novel in its estimation of pediatric-specific mortality and morbidity in the modern era of antibiotics and MRI. This study is limited by its retrospective nature, variation in treatment 
Figure $3 \quad$ Summary of literature review

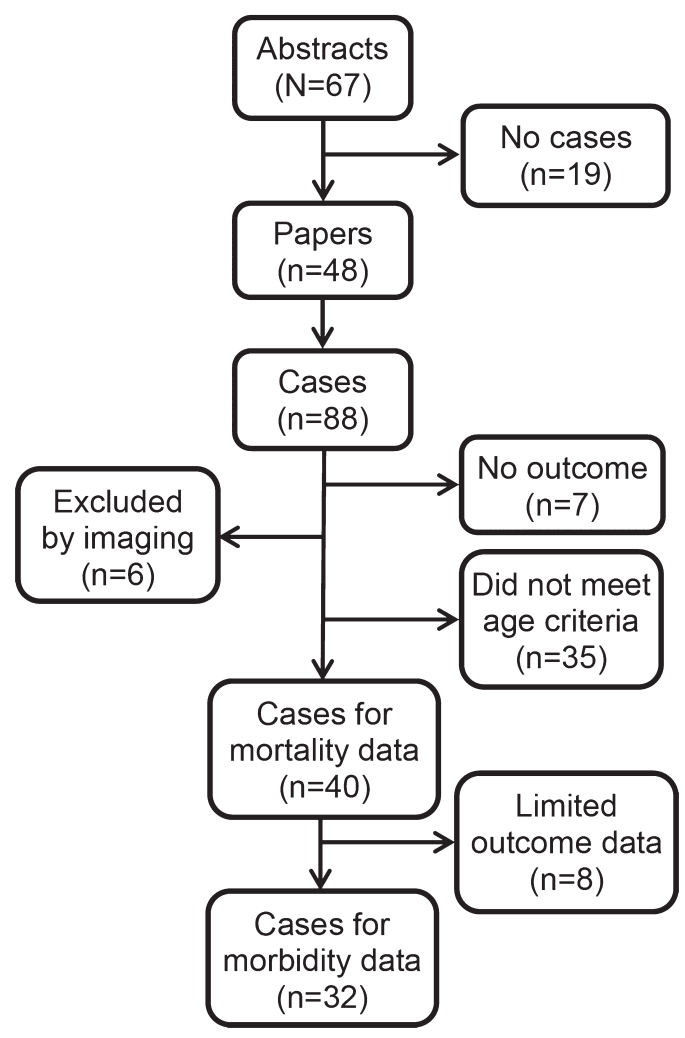

among practitioners, and variability of follow-up duration and data. The review of cases in the literature is limited by publication bias and by the high number of excluded patients due to unavailable information. It is unclear if these limitations would skew the results of this study in favor of more positive or negative outcomes.

As previously established, prompt recognition of the condition and definitive treatment is the key to limiting the complications of CST. ${ }^{18}$ In our singlecenter cohort study, the very high frequency of positive outcomes may be related to several factors,

\begin{tabular}{|c|c|c|c|}
\hline \multirow{2}{*}{$\begin{array}{l}\text { Table } 2 \\
\text { Organism }\end{array}$} & \multicolumn{3}{|c|}{$\begin{array}{l}\text { Microorganisms isolated in cases of } \\
\text { cavernous sinus thrombosis }\end{array}$} \\
\hline & $\begin{array}{l}\text { No. (\%) of } \\
\text { cases, } \\
n=52\end{array}$ & $\begin{array}{l}\text { Morbidity, } \\
n(\%) \\
n=44\end{array}$ & $\begin{array}{l}\text { Mortality, } \\
\text { n (\%), } \\
n=52\end{array}$ \\
\hline $\begin{array}{l}\text { Staphylococcus } \\
\text { aureus }\end{array}$ & 11 (21) & 2/9 (22) & 0 \\
\hline $\begin{array}{l}\text { Streptococcus } \\
\text { species }\end{array}$ & $6(12)$ & 2/6 (33) & $1 / 6$ (17) \\
\hline Fungal species & $3(6)$ & $3 / 3(100)$ & 1/3 (33) \\
\hline Fusobacterium & $3(6)$ & $1 / 2(50)$ & 0 \\
\hline Other & $4(8)$ & $1 / 4$ (25) & 0 \\
\hline Culture negative & 18 (35) & 3/18 (17) & 0 \\
\hline No culture data & 7 (13) & $2 / 2$ (100) & $2 / 7$ (29) \\
\hline
\end{tabular}

including early recognition and aggressive therapy with surgery, antibiotics, and anticoagulation. No guidelines exist directing management of CST, but these outcomes suggest that an aggressive approach should be considered for all patients and deserves further evaluation. This treatment approach should include a combination of IV antibiotics, surgical intervention directed at the infectious precipitant, and consideration of anticoagulation.

CST was historically a clinical diagnosis, ${ }^{19}$ but current medical practice requires imaging for diagnosis. Contrast-enhanced $\mathrm{CT}^{20}$ and contrast-enhanced $\mathrm{MRI}^{19}$ have been found to be the most sensitive imaging modalities and are considered the gold standard in diagnosing CST. Our experience in the 12 children treated at the Children's Hospital of Philadelphia recapitulates these findings, and found $100 \%$ sensitivity of contrast-enhanced head CT or MRI, compared with $0 \%$ for noncontrast. We particularly found 3D T1 precontrast and postcontrast imaging valuable in detecting and characterizing thrombus within the cavernous and dural venous sinuses.

Previous studies have not formally examined the utility of various types of magnetic resonance venography (MRV) in the diagnosis of CST, although cases of abnormal MRVs in CST have been noted. ${ }^{21}$ The 8 TOF MRVs performed in our cohort did not, in any case, provide a diagnosis. This is likely related to the slow and turbulent flow within the cavernous sinuses, making this relatively small area difficult to characterize using this imaging technique. The presence of IV contrast on CT or MRI allows filling of the venous caverns and allows detection of small filling defects caused by clot. As such, noncontrast TOF MRV has a limited role in CST. The utility of other forms of MRV were not investigated. CT venography, which is essentially a form of contrast head CT, is an alternative diagnostic tool as well.

Our cohort identified a low frequency of superior ophthalmic vein thrombosis in association with CST $(25 \%)$. This is likely secondary to the high frequency of sinusitis as the predisposing etiologic factor in our cases. Through communication with the facial vein, the superior ophthalmic vein contains drainage of the "danger triangle" of the face, including the medial angle of the eyes, nose, and perioral region. Additionally, it drains the region of the frontal sinuses. When CST is secondary to facial acne, facial cellulitis, orbital cellulitis, or frontal sinusitis, it is likely due to bacterial invasion via the superior ophthalmic vein. In these circumstances, the vein is more likely to exhibit evidence of thrombophlebitis. It is likely that our cohort found fewer instances of superior ophthalmic vein thrombosis due to the high incidence of CST due to sphenoidal sinusitis. 
Once CST is identified, empiric antimicrobial therapy should be initiated. The causative agents in our series were comparable to previously published findings with a high frequency of Staphylococcus, Streptococcus, and anaerobic species cultured. In our cohort, empiric antibiotics against these agents usually constituted vancomycin, a cephalosporin, and metronidazole. Based on the high morbidity associated with fungal infection, strong consideration should be given to antifungal agents in at-risk populations. These include children who are immunocompromised, have diabetes mellitus, or are chronically on steroids or antibiotics. Antifungals should also be strongly considered in cases that are refractory to first-line therapy.

Surgical intervention directed against the infection precipitating CST should be planned early in the treatment course. Acute bacterial sinusitis with a complication such as CST is an indication for surgical intervention. $^{22}$ In our cohort, surgical intervention primarily consisted of functional endoscopic sinus surgery. Outcomes between children who underwent surgery vs those who did not did not seem to differ; however, it is unlikely that these groups are equivalent. In many of the reviewed cases, surgery was offered when recovery was delayed or the patient worsened. It is unclear if early surgical intervention would have prevented these complications and warrants further investigation.

Similar challenges exist in analyzing outcomes associated with anticoagulation. Bhatia and Jones ${ }^{23}$ previously reviewed the controversy regarding the use of anticoagulants in CST, and there remains no consensus on the issue. In our cohort, the populations were not equivalent: all children who were anticoagulated had narrowing of their internal carotid arteries. Additionally, the 2 children who had septic thromboses of the internal jugular vein were anticoagulated. In other cases in the literature review, anticoagulation was utilized due to the concurrent presence of cerebral venous sinus thrombosis. Despite these variations in population, there was no difference in outcomes. There were no bleeding complications seen in our cohort or in the literature review, suggesting at minimum that anticoagulation in this population is safe.

Multiple previous case reports have noted reversible narrowing of the ICA in association with CST..$^{24,25}$ Our cohort establishes that this is a common occurrence. Every patient who had narrowing of the ICA during the acute phase of the illness had an improved appearance on follow-up imaging, and for $70 \%$ of cases the narrowing resolved within 6 months. ICA narrowing is of unclear clinical significance, but raises concern for the potential for arterial ischemic stroke.

With prompt recognition and treatment, the prognosis of CST in children is better than
Yarington's ${ }^{8}$ previous findings in adults in 1977 , which remain the most recent estimates of CSTspecific morbidity and mortality. As MRI has become more rapidly available, it is likely that the diagnosis is being made sooner in the clinical course, driving better clinical outcomes. Morbidity can result from injury to any of the contents of the cavernous sinus: cranial nerves III, IV, V1, V2, and VI, as well as the internal carotid artery. Clot propagation may result in thrombosed ophthalmic veins and retinal infarction, or may propagate into the deeper venous system and cause petrosal sinus, sigmoid sinus, or internal jugular vein thrombosis. Without rapid, definitive treatment, CST remains a fatal disease. Given the rarity of this condition, it remains a challenge to study. At this time, it remains unclear what the optimal management is in terms of anticoagulant use, timing, selection, and duration. Additionally, the role of surgery and its timing would benefit from future investigation.

\section{AUTHOR CONTRIBUTIONS}

Dr. Smith performed the data abstraction, analyzed all data, did the literature review, and wrote the manuscript. Drs. Vossough and Vorona performed the review of radiology records, reviewed the images of all patients, and edited the manuscript. Drs. Beslow and Ichord recruited patients from the clinical stroke service and edited the manuscript. Dr. Licht served as mentor to the project, provided guidance, and performed edits on all drafts of the manuscript.

\section{STUDY FUNDING}

No targeted funding reported.

\section{DISCLOSURE}

The authors report no disclosures relevant to the manuscript. Go to Neurology.org for full disclosures.

Received January 6, 2015. Accepted in final form May 6, 2015.

\section{REFERENCES}

1. Morelli N, Rota E, Sacchini D, et al. Lemierre syndrome: more than "the forgotten disease." Neurology 2013;81: 1179-1180.

2. Clarke M, Enuh H, Saverimuttu J, Nfonoyim J. Streptococcus group $\mathrm{C}$ meningitis with cavernous sinus thrombosis. Infect Drug Resist 2013;6:79-81.

3. Dyer SR, Thottam PJ, Saraiya S, Haupert M. Acute sphenoid sinusitis leading to contralateral cavernous sinus thrombosis: a case report. J Laryngol Otol 2013;127: 814-816

4. Pollock TJ, Kim P, Sargent MA, Aroichane M, Lyons CJ, Gardiner JA. Ophthalmic complications of otitis media in children. J AAPOS 2011;15:272-275.

5. Okamoto H, Ogata A, Kosugi M, Takashima H, Sakata S, Matsushima T. Cavernous sinus thrombophlebitis related to dental infection: two case reports. Neurol Med Chir 2012;52:757-760.

6. Senthilkumaran $S$, Balamurugan $N$, Sweni $S$, Thirumalaikolundusubramanian P. Cavernous sinus thrombosis following bee sting. Int J Crit Illn Inj Sci 2011;1:167-168. 
7. Vidhate MR, Sharma P, Verma R, Yadav R. Bilateral cavernous sinus syndrome and bilateral cerebral infarcts: a rare combination after wasp sting. J Neurol Sci 2011;301:104106.

8. Yarington CT. Cavernous sinus thrombosis revisited. Proc R Soc Med 1977;70:456-459.

9. Yarington CT. The prognosis and treatment of cavernous sinus thrombosis: review of 878 cases in the literature. Ann Otol Rhinol Laryngol 1961;70:263-267.

10. Ebright JR, Pace MT, Niazi AF. Septic thrombosis of the cavernous sinuses. Arch Intern Med 2001;161:26712676.

11. Ali SM, Ahmed SH. Cavernous sinus thrombosis in children. J Trop Pediatr 1992;38:194-195.

12. Visudtibhan A, Visudhiphan P, Chiemchanya S. Cavernous sinus thrombophlebitis in children. Pediatr Neurol 2001;24:123-127.

13. Ryan M, Yeo S, Maguire A, et al. Rhinocerebral zygomycosis in childhood acute lymphoblastic leukaemia. Eur J Pediatr 2001;160:235-238.

14. Saetang S, Preechawai P, Hirunpat S. Retrograde cavernous sinus thrombosis and orbital cellulitis secondary to meningitis in immunocompetent child. J Med Assoc Thai 2012;95:1485-1488.

15. Schuknecht B, Simmen D, Yüksel C, Valavanis A. Tributary venosinus occlusion and septic cavernous sinus thrombosis: CT and MR findings. AJNR Am J Neuroradiol 1998;19:617-626.

16. Sasindran V, Ravikumar A, Senthil. Orbital apex syndrome in a child. Indian J Otolaryngol Head Neck Surg 2008;60:62-65.
17. Gelston CD, Durairaj VD, Simoes EA. Rhino-orbital mucormycosis causing cavernous sinus and internal carotid thrombosis treated with posaconazole. Arch Ophthalmol 2007;125:848-849.

18. Desa V, Green R. Cavernous sinus thrombosis: current therapy. J Oral Maxillofac Surg 2012;70:2085-2091.

19. Berge J, Louail C, Caillé JM. Cavernous sinus thrombosis diagnostic approach. J Neuroradiol 1994;21:101-117.

20. de Slegte RG, Kaiser MC, van der Baan S, Smit L. Computed tomographic diagnosis of septic sinus thrombosis and their complications. Neuroradiology 1988;30:160165.

21. Ozsvath RR, Casey SO, Lustrin ES, Alberico RA, Hassankhani A, Patel M. Cerebral venography: comparison of CT and MR projection venography. AJR Am J Roentgenol 1997;169:1699-1707.

22. Stokken J, Gupta A, Krakovitz P, Anne S. Rhinosinusitis in children: a comparison of patients requiring surgery for acute complications versus chronic disease. Am J Otolaryngol 2014;35:641-646.

23. Bhatia K, Jones NS. Septic cavernous sinus thrombosis secondary to sinusitis: are anticoagulants indicated? A review of the literature. J Laryngol Otol 2002;116:667-676.

24. Madhusudhan KS, Kandpal H, Ahuja J. Cavernous sinus thrombophlebitis causing reversible narrowing of internal carotid artery. Neurol India 2009;57:102-103.

25. Hoshino C, Satoh N, Sugawara S, Kuriyama C, Kikuchi A, Ohta M. Septic cavernous sinus thrombosis complicated by narrowing of the internal carotid artery, subarachnoid abscess and multiple pulmonary septic emboli. Intern Med 2007;46:317-323.

\section{How Do YOU Compare? Access New Neurology Compensation and Productivity Report}

The AAN's 2015 Neurology Compensation and Productivity Report is now available. Based on data from more than 1,300 neurologists and neurology practice managers, this is the most recent and reliable information on the neurology profession.

The Neurology Compensation and Productivity Report is a powerful, versatile tool that can help you:

- Compare and customize your individual practice-related data with your colleagues at national and local levels

- Determine if you are being paid fairly relative to your peers

- Use the data in contracting with payers and demonstrating your value

- Discover fair market value based on your subspecialty, region, and practice type

- Create charts and graphs and download them right to your desktop

- Assess patient and practice management principals and implement efficiencies that ultimately can help improve the quality of patient care

Learn more at $A A N$.com/view/2015NeuroReport. 